\title{
USO Y CREDIBILIDAD DE FUENTES PERIODÍSTICAS 2.0 EN PORTUGAL Y ESPAÑA
}

\author{
João Canavilhas y Begoña Ivars-Nicolás
}
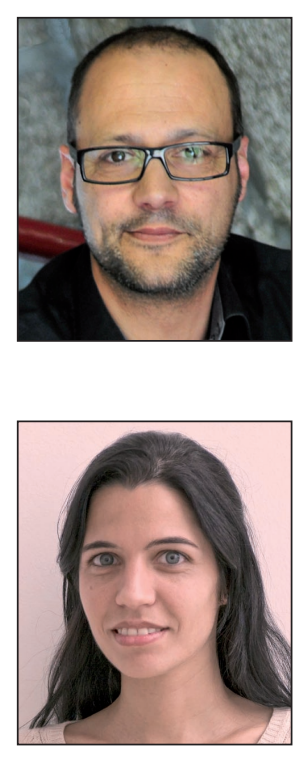

João Canavilhas, licenciado en comunicação social por la Universidade da Beira Interior, UBI (Portugal) y doctor por la Universidad de Salamanca (España), es profesor en la UBI donde imparte clases de webperiodismo, periodismo radiofónico e infografía multimedia. Además es director del Mestrado em Jornalismo, vicepresidente del Centro de Investigación Labcom-UBI y director del diario online $U R B I$, el primer periódico universitario portugués en la Web.

Universidade da Beira Interior Faculdade de Artes e Letras Av. Marquês d'Ávila e Bolama 6200001 Covilhã, Portugal jc@ubi.pt

Begoña Ivars-Nicolás es profesora de periodismo en la Universidad Miguel Hernández (UMH) de Elche y de comunicación audiovisual en el Centro de Estudios Ciudad de la Luz adscrito a esta misma universidad. Doctora en antropología social, centra sus investigaciones en los medios de comunicación en la Red y en la repercusión de las TIC en la docencia. Coedita la revista digital de comunicación audiovisual Croma y codirige las ediciones del CICAP Congreso Internacional de Comunicación Audiovisual y Publicidad de la UMH.

Universidad Miguel Hernández Av. de la Universidad, $\mathrm{s} / \mathrm{n}$ 03202 Elche (Alicante), España bivars@umh.es

\section{Resumen}

Internet ha cambiado las rutinas de producción en el periodismo. La búsqueda de información ha sido una de las áreas más afectadas, con la aparición de fuentes 2.0 como blogs y redes sociales. A pesar del aparente valor añadido de estas nuevas fuentes de información, ¿qué utilización práctica les dan los periodistas portugueses y españoles y qué credibilidad les atribuyen? Se intenta responder a estas dos cuestiones, definiendo las aplicaciones que pueden considerarse fuentes 2.0 y en qué medida los periodistas las estiman creíbles y fiables. Los resultados muestran que los periodistas de ambos países siguen utilizando internet diariamente para buscar material para su trabajo, documentarse, recibir y enviar información y verificarla, pero aún confían poco en las fuentes 2.0 .

\section{Palabras clave}

Internet, Periodismo, Fuentes de noticias, Fuentes de información, Credibilidad, Web, Web 2.0.

Title: Use and credibility of news sources 2.0 in Portugal and Spain

\begin{abstract}
Internet has changed news making. The search for information has been one of the most affected areas, with the emergence of news sources 2.0, such as blogs and social networks. Despite the apparent value of these new sources of information, what practical use and credibility do Portuguese and Spanish journalists give to these sources? In this paper we define what kind of tools can be considered " 2.0 sources" and the ways in which these journalists consider them credible and reliable. The results show that the journalists from both countries are using the internet daily to find material for their work, get factual information, receive and send information and verify it, but still have little confidence in the 2.0 sources.
\end{abstract}

\section{Keywords}

Internet, Journalism, News sources, Information sources, Credibility, Web, Web 2.0.

Canavilhas, João; Ivars-Nicolás, Begoña. "Uso y credibilidad de fuentes periodísticas 2.0 en Portugal y España". El profesional de la información, 2012, enero-febrero, v. 21, n. 1, pp. 63-69. 


\section{Introducción}

En el origen de toda noticia existe una fuente de información. Pero ¿de qué hablamos cuando nos referimos a fuentes de información? De acuerdo con Herbert Gans, en el contexto del periodismo, fuentes son "los actores que los periodistas observan o entrevistan, incluidos los entrevistados que hablan por los medios o que son citados en artículos de revista, y también los que sólo suministran información de antecedentes o sugieren temas y narraciones" (2004, p. 80).

Los actores observados o entrevistados a los que se refiere Gans asumen diferentes posiciones en la taxonomía de fuentes según sea su implicación con el hecho objeto de la noticia. Si tomamos como punto de partida la clasificación más generalista, las fuentes pueden ser oficiales o no oficiales, y es en este campo donde internet ha introducido cambios significativos, con nuevas formas de buscar información, localizar expertos y contactar con fuentes no oficiales (Pavlik, 2001).

En este trabajo hacemos un recorrido por los cambios en las rutinas periodísticas de los profesionales ibéricos, sobre todo en la fase de recoger temas e informaciones para noticias. Seguidamente definimos el término fuentes 2.0, integrándolo en el concepto grupal de web 2.0. Para terminar el marco teórico, se discute la credibilidad de las fuentes de información en la Web y los criterios que emplean los usuarios para seleccionar la información más creíble. En el estudio empírico se presentan los resultados de una encuesta a periodistas de España y Portugal sobre las fuentes 2.0 que utilizan y el grado de credibilidad que les reconocen.

\section{Internet en las rutinas de los periodistas: Portugal y España}

Desde los años 90 del siglo pasado la práctica profesional del periodismo ha experimentado notables cambios como consecuencia del uso de internet en las redacciones. El aspecto más visible ha sido el nacimiento de periódicos online, pero la Red también ha transformado las rutinas de trabajo. La búsqueda de material para reportajes, la recogida de informaciones para complementar las noticias y el contacto con las fuentes han sufrido importantes alteraciones. La integración de aplicaciones online en el trabajo diario ha acelerado el proceso de producción de noticias.

\subsection{Portugal}

La primera investigación sobre internet y periodistas realizada en Portugal (Bastos, 1998) reveló que éstos lo utilizaban para buscar información (100\%), obtener datos más actualizados (93,5\%), leer y enviar correo electrónico $(87,5 \%)$ e identificar expertos para aclarar dudas relacionadas con el material de sus noticias $(62,5 \%)$.

Seis años después, Canavilhas (2004) repitió la encuesta obteniendo cifras similares en búsqueda de información $(97,5 \%)$, obtención de datos actualizados $(83,9 \%)$ y utilización del correo electrónico $(92,6 \%)$, pero distinta en la identificación de expertos (30,9\%). El cambio podría estar justificado porque en este estudio existía la opción alternativa "Contactar fuentes" que recibió el 53,5\% de las preferen- cias. Las aplicaciones más utilizadas eran la Web $(97,5 \%)$, el correo electrónico $(93,8 \%)$, los foros $(18,5 \%)$, los grupos de noticias $(16 \%)$ y los chats $(9,9 \%)$. Se preguntaba igualmente por la percepción que tenían los periodistas de la fiabilidad de la información de internet: el 32\% la consideró fiable, el $37 \%$ se mostraba sin opinión y el $21 \%$ no la consideraba creíble.

En 2006 Obercom realizó un estudio más detallado en el que participaron 341 periodistas de 14 medios de comunicación portugueses. El 99,4\% de los encuestados valoró internet como "importante" ${ }^{1}$ para el ejercicio de sus funciones profesionales. En general, los más jóvenes atribuyeron más valor al papel de este medio en sus rutinas diarias. El 99,7\% lo utilizaba en su profesión, casi todos con un uso diario $(99,4 \%)$, y el $87 \%$ manejaba diferentes aplicaciones online más de cinco veces al día.

Con referencia a las aplicaciones de la Red, el 99,1\% consideró los sitios web "importantes" para su trabajo y el correo electrónico presentó valores muy similares $(98,8 \%)$. En el análisis de este medio como fuente, el 96,7\% consideró las búsquedas online "importantes". Subrayando este hecho, el $85,2 \%$ respondió que la utilizaba diariamente. El uso de grupos de noticias como fuente fue poco valorada: solamente el $22 \%$ la consideró "importante", una cifra inferior a los $30,5 \%$ de las listas de correo y los $31,4 \%$ de los blogs.

En 2010 Obercom ha llevado a cabo otro estudio con el propósito de "obtener una percepción de los valores, prácticas y actitudes que caracterizan la profesión" (2010, pág. 7). En un capítulo dedicado a las fuentes, internet destaca una vez más como primera posibilidad de recogida de información $(51,9 \%)$, superando en más del doble a la siguiente fuente, la prensa $(24,6 \%)$. Los periodistas de prensa son los que más optan por internet $(67,1 \%)$, seguidos por los de radio $(52,2 \%)$ y los de televisión $(40,6 \%)$. La encuesta igualmente ha indagado sobre el tipo de aplicaciones que utilizan los encuestados: de las opciones propuestas, los lectores de feeds son la fuente más utilizada $(30,2 \%)$, seguido de los UGC (user-generated content) $(29,2 \%)$, los blogs $(25,5 \%)$, el crowdsourcing $(22,6 \%)$ y el podcast $(18,9 \%)$. Hay que señalar que varias de estas categorías se solapan.

\subsection{España}

Entre los trabajos que abordan el uso de internet en las redacciones españolas o su credibilidad, se encuentra el realizado por AccesoGroup (2002) que encuestó a 616 periodistas. Los datos obtenidos mostraron que los profesionales la empleaban como fuente de información (97\%): el 49\% usaba la Red para todas sus noticias y el $48 \%$ sólo en la mitad de sus trabajos. El $96 \%$ le atribuyó mucha credibilidad; el $60 \%$ la consideró una fuente de información y el $40 \%$ una fuente de documentación: entre los dos grupos, los primeros dieron mayor credibilidad a internet.

El Col/legi de Periodistes de Catalunya estudia desde 1992 el perfil del periodista mediante encuestas. En un estudio de 2002, Masip concluyó que el $85 \%$ de los profesionales catalanes utilizaban internet diariamente: correo electrónico (75\%), cibermedios, buscadores y webs institucionales. Sin embargo, el $95 \%$ de los periodistas consideraba la Red como una fuente de información carente de confianza de- 
bido a la dificultad en verificar la autoría y a la falta de credibilidad, prefiriendo sistemas tradicionales como el cara a cara, el teléfono y la prensa. En 2005, Masip reforzó su estudio cuantitativo de 2002 con la observación de campo de tres redacciones catalanas obteniendo resultados similares que además fortalecieron el uso de la Red. Los periodistas catalanes encontraron ventajoso usar internet para documentarse sobre noticias ya previstas, de largo recorrido, sustituyendo los centros de documentación de los medios y fomentando el "periodismo de datos" para complementar y enriquecer las noticias.

En 2009 se llevó a cabo otra investigación en el País Vasco y Navarra (Díaz-Noci et al., 2010) y los datos obtenidos poco distaban de los recogidos en Catalunya. En cuanto al origen de las noticias, los profesionales vascos y navarros no otorgaron fiabilidad a internet. Opinaron que abundaba la información de mala calidad $(38,7 \%)$, era difícil establecer la credibilidad de un sitio web $(25,8 \%)$, así como su autoría $(14,5 \%)$.

En 2011 se ha publicado la cuarta edición del Estudio sobre periodismo digital 2011 llevado a cabo por Oriella PR Network. Esta investigación internacional, realizada a 475 periodistas de 15 países, incluido España, ha analizado por primera vez el uso de los medios sociales para buscar y comprobar información en las redacciones. Los resultados han concluido que en España un tercio utiliza redes sociales como Twiter (31\%) y Facebook (28\%) para buscar y contrastar noticias. También acceden a blogs tanto de autores conocidos (31\%) como desconocidos (15\%). No obstante, los profesionales españoles consideran estas fuentes de información no fiables.

Por fuentes 2.0 entendemos los suministradores de información que lo hacen por libre y espontánea voluntad del autor, sea un individuo o un grupo, utilizando herramientas colaborativas

\section{Fuentes $\mathbf{2 . 0}$}

Por web 2.0 se entiende la Web colaborativa, es decir, donde los lectores son más que consumidores pasivos, contribuyendo con su conocimiento al enriquecimiento de la misma. En este concepto, que tiene una vertiente más tecnológica y otra más social, la Web se aleja del patrón inicial de plataforma de distribución de información y se sitúa en el eje del paradigma descentralizado donde la participación de los interesados es el combustible del medio.

En el campo de las fuentes de información periodísticas ocurre lo mismo, siendo posible dividirlas en dos grupos: las propias de la primera web, de naturaleza más informacional, con predominio de las oficiales, y las fuentes de la web 2.0, basadas en la contribución de la inteligencia colectiva y, en ese sentido, son predominantemente no oficiales.

En su concepto original, inteligencia colectiva es la forma en que "las organizaciones sociales (...) se agrupan para com- partir y colaborar, para encontrar una ventaja individual y colectiva mayor que si cada participante hubiese permanecido solo" (Noubel, 2006, p. 5). Esta inteligencia colectiva inicial está condicionada por dos factores: el número de participantes y el espacio, ya que su eficacia depende de que el grupo tenga un número adecuado de participantes y que estos estén físicamente cercanos. Las dos limitaciones han sido superadas con internet, gracias a que las aplicaciones informáticas "acercan" virtualmente a los lectores, posibilitando el trabajo comunitario, independientemente del número de participantes. En esta nueva realidad, Noubel propone que inteligencia colectiva sea "la capacidad de un grupo de personas para colaborar en orden a decidir sobre su propio futuro y alcanzarlo en un contexto complejo" (2006, p. 16). Esta capacidad de colaboración (vertiente social de la web 2.0) es potenciada por las nuevas aplicaciones informáticas (vertiente tecnológica) y da lugar a nuevas fuentes de conocimiento online que, sobre todo en el caso de las grupales, son lo que llamamos fuentes de información 2.0.

Manuel Pinto (1999) hace un recorrido por las diferentes taxonomías de fuentes e identifica siete tipos, en función de su naturaleza, origen, duración, área geográfica, implicación en los hechos, actitud de cara al periodista, identificación y estrategia de actuación. En el actual ecosistema mediático donde internet juega un papel central, proponemos un nuevo tipo basado en el rol del profesional en la manera cómo utiliza en su trabajo las nuevas aplicaciones online. Esta taxonomía nos parece acertada porque se ubica en una variable propia de los medios online, la interacción, sea por iniciativa del profesional o del lector.

- Fuentes push (el usuario es "empujado" por la información): se acercan a las propiedades de las fuentes tradicionales. El rol del periodista es más pasivo porque se limita a recibir informaciones de organizaciones, grupos o personas. Esa información puede ser recibida mediante una inscripción previa en un grupo/feed, o porque las relaciones públicas de esa organización añadieron su contacto al grupo. En este caso hablamos de aplicaciones como el correo electrónico, los grupos de usuarios, o los lectores de feeds.

- Fuentes pull (el usuario tira de la información): el profesional toma la iniciativa de buscar la información porque sabe lo que pretende y realiza búsquedas por temas opalabras clave. Las aplicaciones típicas de este tipo de investigación son motores de búsqueda, webs, foros, chats, wikis, blogs o redes sociales.

Aunque las redes sociales y los blogs son las fuentes 2.0 más utilizadas en las rutinas periodísticas, los profesionales consideran más fiables los repositorios y los wikis

La taxonomía propuesta facilita la separación de las fuentes en dos grupos: las que son simplemente online y las que además pueden ser consideradas 2.0. ¿Cuáles son las particularidades o cualidades que marcan la diferencia? 
Por fuentes 2.0 entendemos los suministradores de información que lo hacen por libre y espontánea voluntad del autor, sea un individuo o un grupo, utilizando las herramientas colaborativas.

a)Blogs y microblogs: nacidos como diarios online, se han transformado en fuentes de opinión alternativas muy utilizadas por los periodistas. Su sencillez de edición ha hecho posible que meros ciudadanos y expertos sin cabida en los medios tradicionales hayan creado una vasta oferta de opinión alternativa muy especializada en determinadas disciplinas. Al utilizar abundante hipertexto en los posts, los blogueros crean una red compleja entre blogosfera y mediasfera, montando un sistema informativo contextualizado que materializa un ejemplo de inteligencia colectiva.

b) Redes sociales: aunque en una primera fase no se hayan asumido como fuentes de información por tratarse de simples aplicaciones personales, desde su utilización por Barack Obama en las elecciones presidenciales norteamericanas de 2008 y a raíz del enorme crecimiento de Facebook, se han transformado en un novedoso palco para las figuras públicas. En este caso, más que la opinión anónima o de expertos, las redes sociales funcionan como una forma de acceso directo y alternativo a las fuentes.

c) Foros o listas de correo: no son una novedad, pero el nacimiento de aplicaciones user friendly ha aumentado su éxito. El posterior nacimiento de las redes sociales, con la posibilidad de constituir grupos, y los wikis, han reducido su impacto. Aun así, hay millones de comunidades muy especializadas donde es posible recoger información fuera de los circuitos principales.

d)Chats: son una forma de contacto interpersonal o grupal, por audio o vídeo, que utiliza internet como canal.

e)Wikis: están hechos con un programa que posibilita que varias personas editen un mismo documento. Han dado lugar a crear enciclopedias colaborativas, siendo Wikipedia la más conocida. En sus primeros años, la edición de textos era muy accesible, por lo que se han creado muchas páginas falsas o con informaciones incorrectas, lo que manchó su credibilidad como fuente de información. En la actualidad, hay grupos de voluntarios (wikipedistas) que controlan las actualizaciones.

f) Repositorios multimedia: en este grupo de fuentes se integran las bases de datos de fotos, sonidos y vídeos. Flickr, Soundcloud o Youtube se encuentran entre los más utilizados por los medios de comunicación. Su éxito está conectado con la popularización de las grabadoras de vídeo y, más recientemente, de los móviles con capacidad de grabar sonidos, vídeos y fotos.

Los motores de búsqueda no son una fuente directa de información, sino un intermediario que facilita encontrarla.

\section{Fuentes y credibilidad en el contexto digital}

En 2002, Online News Association (ONA) llevó a cabo un estudio sobre noticias online y credibilidad. Un dato sobresaliente es que el $91,5 \%$ de los encuestados consideró más creíbles los sitios web de organizaciones informativas tradicionales. Esta orientación se confirmó en otros estudios (Cassidy, 2007) donde la credibilidad percibida por los lec- tores siempre asocia el medio online a su versión tradicional. Pero ¿qué ocurre cuando no hay un referencial perceptible, es decir, cuando el usuario que busca información no conoce el actor que está detrás del medio?

Metzger (2007) hace una revisión bibliográfica en autores de diferentes áreas e identifica cinco criterios que los usuarios emplean para evaluar la credibilidad de una información en internet:

- exactitud: ausencia de errores y posibilidad de confirmar la información en otro soporte;

- autoridad: información sobre el autor, afiliaciones y reconocimiento de la comunidad;

- objetividad: identificación de los propósitos del sitio o utilización de un lenguaje persuasivo;

- actualización periódica: ritmo de oferta de informaciones;

- cobertura/alcance: profundidad y amplitud de la información ofrecida.

Si la exactitud y la objetividad son criterios conectados con la credibilidad, independientemente de que la información original sea digital o tradicional, los criterios de autoridad, actualización y cobertura nos conducen a fenómenos grupales de colaboración. En ese sentido, la credibilidad de las fuentes online tendría una fuerte conexión con las características de las herramientas 2.0 (vertiente tecnológica), que son el motor de la inteligencia colectiva (vertiente social) enunciada por Noubel (2006). Esto es, la información irrumpe desde un hecho casi insignificante o una información incompleta, y por la fuerza de la colaboración existente entre los expertos online y de las posibilidades de los instrumentos, crece hasta tener un valor informativo para los periodistas.

El criterio de autoridad de la fuente se fundamenta en su forma de legitimación, es decir, en el proceso que permite su reconocimiento en la comunidad online. Por ejemplo, los blogs se legitiman entre pares: el ranking de Technorati es una forma de legitimación interna que cruza el número de visitas, los enlaces dirigidos a los blogs y el prestigio de quien publica esos enlaces. En el caso de Twitter, importa más el contenido compartido que los atributos de los usuarios (Asur et al., 2011), lo que reduce el peso del actor y subraya el del instrumento comunitario.

Para evaluar la credibilidad de una información en internet se emplean cinco criterios: exactitud, autoridad, objetividad, actualización y profundidad

En el caso de la actualización permanente hay un vínculo con las características del medio (internet), pero también con la percepción de que los temas actuales y más atractivos suscitan una mayor participación. En este caso, el mejor ejemplo son los foros o listas, espacios frecuentados por especialistas en un determinado tema que mantienen una permanente vigilancia sobre la calidad de la discusión.

Por último, la cobertura nos lleva una vez más a espacios de inteligencia colectiva. Es el caso de los wikis, donde la infor- 
mación no tiene el ritmo de actualización de los foros, pero presenta semejante nivel de profundidad y de vigilancia.

Para otros autores (O’Keefe, 2002; David; Glore, 2010), el diseño y la usabilidad son igualmente factores que influyen en la credibilidad de la información online. En ese sentido las herramientas de la web 2.0, diseñadas para una fácil utilización, son una referencia de usabilidad y, en consecuencia, la información que se difunda en ellas tiene potencial para generar mayor percepción de credibilidad.

\section{Metodología y procedimiento}

Este estudio intenta conocer el uso por parte de los periodistas portugueses y españoles de fuentes 2.0 y el grado de credibilidad que les reconocen. Se elaboró un cuestionario online realizado entre el 27 de julio y el 17 de agosto de 2011 a periodistas de diferentes medios de comunicación social de Portugal y España. La distribución del enlace al cuestionario se hizo de dos formas: por correo electrónico a los periodistas solicitando que rellenaran en documento online, o contactando con el director del medio para que difundiera el enlace desde la red interna del mismo.

http://www.in2web.es/fuentes20

Se optó por enviar el enlace a medios de comunicación con fuerte presencia en internet por considerar que serían los que más utilizan las fuentes 2.0 .

En Portugal se envió a periodistas de los más importantes:

- diarios: Correio da manhã, Jornal de notícias, Diário de notícias, Público e I;

- semanarios: Expresso y Sol;

- radios informativas: Antena 1 y TSF;

- televisiones: RTP, SIC y TVI.

En España se escogieron los medios de comunicación nacional más visitados según datos del ranking Alexa en la fecha de realización del estudio:

- televisiones: Telecinco, RTVE, Cuatro y Antena3;

- radios nacionales: Cadena SER, Los 40 principales, Intereconomía, Cope, Onda cero, Cadena 100, Punto radio, Europa FM, y Catalunya radio;

- prensa escrita nacional: El país, El mundo, 20 minutos, $A B C$, La vanguardia, Xornal Galicia y La razón;

- prensa digital: Libertad digital, Minuto digital y Estrella digital.

En total se han obtenido 202 cuestionarios válidos, 58 de Portugal y 144 de España. No se apuró la tasa de retorno porque el estudio coincidió con el período de vacaciones y en muchos casos las redacciones funcionaban con menos personal del habitual.

La mitad de los periodistas ibéricos encuestados realiza su labor en prensa impresa, alrededor de una cuarta parte lo hace en televisión $(23,7 \%$ ) y radio $(23,2 \%)$, y apenas un $20 \%$ en cibermedios. Por país, los periodistas portugueses más representados son los de prensa $(46,6 \%)$, seguidos de los de radio $(27,6 \%)$, televisión $(22,4 \%$ ) y cibermedios $(17,2 \%)$. En cuanto a los españoles, el 56,9\% de los encuestados trabaja en prensa impresa, el $25 \%$ en televisión, el $19,4 \%$ en internet y el $18,8 \%$ en radio. Un factor interesante es que el $8,6 \%$ de los profesionales lusos y el $18 \%$ de los españoles afirman trabajar en dos medios a la vez.

El cuestionario constaba de cinco bloques de preguntas, pero para este artículo se han utilizado solamente tres:

- el primero dirigido a conocer datos personales del profesional y sus rutinas con respecto al uso de la Red en su labor diaria, con cuestiones cerradas de opción múltiple;

- el segundo grupo buscaba medir la percepción de credibilidad que los periodistas depositan en las informaciones procedentes de las llamadas fuentes 2.0. Para evaluarlo, se utilizó la escala de Likert desde 1 (nada creíble) a 5 (muy creíble);

- el tercer bloque de cuestiones buscaba saber qué tipo de fuentes 2.0 había estado en el origen de noticias publicadas por los encuestados.

\section{Resultados}

En 2011 la Red se utiliza diariamente en las tareas periodísticas en cualquiera de los cuatro medios. Más del $75 \%$ de los informadores la usa con la finalidad de buscar material para su trabajo, documentarse y verificar la información, contactar fuentes y recibir y enviar información.

Pero ¿cuáles son las principales fuentes o aplicaciones que utilizan en internet para obtener la información?

Los datos obtenidos revelan que las más empleadas en las redacciones portuguesas son los contactos personales (75,9\%), notas de prensa (press releases) $(72,4 \%)$, otros medios de comunicación (72,4\%), motores de búsqueda $(70,7 \%)$, y artículos científicos $(67,2 \%)$. Las redes sociales son usadas por $50 \%$ de los periodistas portugueses, los blogs por $31 \%$, los repositorios por $25,9 \%$ y los wikis por $24,1 \%$. Los foros $(6,9 \%)$ y los chats $(5,2 \%)$ cierran las preferencias.

En España, los periodistas recurren igualmente a contactos personales $(87,5 \%)$, motores de búsqueda $(62,5 \%)$, redes sociales $(62,5 \%)$ y otros medios de comunicación $(56,3 \%)$. Las fuentes que reúnen menos de la mitad en preferencias son wikis $(43,8 \%)$, artículos científicos $(31,3 \%)$, repositorios y blogs ( $25 \%$ cada uno) y, en último lugar, chats y foros (12,5\% cada uno). Es notable la diferencia de uso de algunas fuentes entre ambos países como sucede con los artículos científicos (Portugal: 67,2\%; España: 31,3\%) y los wikis (Portugal: 24,1\%; España: 43,8\%).

Con relación a las fuentes de internet, los resultados totales indican que algo más de la mitad de los periodistas utiliza los motores de búsqueda $(67,3 \%)$ y las redes sociales $(56,3 \%)$. Menos populares son los wikis (34\%), los blogs (28\%), los repositorios $(25,5 \%)$, los chats $(8,9 \%)$ y los foros $(9,7 \%)$.

La parte siguiente del cuestionario valora el nivel de confianza de estas fuentes. Se destaca que alrededor del $20 \%$ de los periodistas ibéricos no sabe qué responder a estas cuestiones. Son los encuestados que utilizan con menos frecuencia la Red como medio de información, documentación y comunicación en su trabajo.

En Portugal ninguna fuente ha obtenido valores de credibilidad por encima del 50\%: más de la mitad opina que los chats $(65,5 \%)$, los foros $(58,6 \%)$, las redes sociales $(53,4 \%)$ y los 


\begin{tabular}{|l|c|c|}
\hline & $\begin{array}{c}\text { Portugal } \\
\%\end{array}$ & $\begin{array}{c}\text { España } \\
\%\end{array}$ \\
\hline Contactos personales & 75,9 & 87,5 \\
\hline Otros medios de comunicación & 72,4 & 56,3 \\
\hline Motores de búsqueda & 70,7 & 62,5 \\
\hline Artículos científicos & 67,2 & 31,3 \\
\hline Redes sociales & 50,0 & 62,5 \\
\hline Blogs & 31,0 & 25,0 \\
\hline Repositorios & 25,9 & 25,0 \\
\hline Wikis & 24,1 & 43,8 \\
\hline Foros & 6,9 & 12,5 \\
\hline Chats & 5,2 & 12,5 \\
\hline
\end{tabular}

Tabla 1. Fuentes más empleadas

blogs $(51,7 \%)$ son fuentes "poco creíbles" o "nada creíbles". Respecto a la credibilidad de los wikis y los repositorios, el $34,5 \%$ los valora fiables, y estas son las únicas fuentes 2.0 con más evaluaciones positivas que negativas.

Entre los españoles hay una fuente 2.0 que destaca por ser considerada "creíble" o "muy creíble" por más de la mitad de los encuestados: son los repositorios (56,3\%). Las redes sociales $(43,8 \%)$ y los wikis $(37,5 \%)$, también presentan más opiniones positivas que negativas. Los chats (75\%), los foros $(43,8 \%)$ y los blogs $(37,5 \%)$ son considerados fuentes "poco creíbles" o "nada creíbles".

Se señala una vez más alguna discrepancia entre periodistas portugueses y españoles, en el sentido de que éstos últimos confían más en las fuentes 2.0. Aunque con valoraciones diferentes, repositorios y wikis están entre las tres fuentes más creíbles para ambos. En el caso español se destaca el valor de credibilidad obtenido por las redes sociales $(43,8 \%)$ comparado con los portugueses (25,9\%).

Si analizamos los resultados conjuntos de periodistas portugueses y españoles, los repositorios (46,2\%), los wikis (36\%), las redes sociales $(34,8 \%)$ y los blogs $(29,4 \%)$ son los instrumentos calificados como "creíbles" o "muy creíbles". No obstante, en ningún caso estas aplicaciones gozan de la confianza de más del $50 \%$ de los encuestados de ambos países.

Con este planteamiento de las fuentes 2.0 como origen de noticias, se preguntó a los periodistas si habían publicado alguna noticia basada en informaciones recogidas en estas plataformas. Las redes sociales son la fuente que más noticias originó $(68 \%)$, con valores muy semejantes entre españoles $(68,8 \%)$ y portugueses $(67,2 \%)$. Los blogs se posicionan en segundo lugar (49,5\%), siendo los portugueses $(55,2 \%)$ los que han recurrido más a ellos que los españoles $(43,8 \%)$. En el caso portugués se trata de una consecuencia de la ligación histórica entre periodistas y blogueros. Los foros ocupan la tercera posición en el origen de noticias $(27,7 \%)$, pero en este caso los españoles $(31,3 \%)$ presentan resultados más altos que los portugueses (24,1\%). Wikis $(24,5 \%)$, repositorios $(20,9 \%)$ y chats $(10,5 \%)$, presentan resultados equilibrados entre los dos países.

Resulta interesante señalar que las redes sociales son consideradas "creíbles" o "muy "creíbles" por solamente el 34,8\% de los periodistas ibéricos, pero el $68 \%$ publicó noticias con origen en informaciones recogidas en esta fuente. La discrepancia es más destacada en el caso de los periodistas portugueses: solamente $25,9 \%$ las considera fiables pero son la fuente 2.0 que más noticias originó (67,2\%). Los blogs, segunda fuente entre las que más noticias originaron en los dos países, también muestra esta discrepancia entre credibilidad percibida $(29,4 \%)$ y utilización en noticias $(49,5 \%)$.

\section{Conclusiones}

La intención de este trabajo es definir las fuentes 2.0 y, esencialmente, conocer el uso que los periodistas hacen de las mismas y el grado de fiabilidad que depositan en ellas.

Se confirma que los periodistas ibéricos, independientemente del medio de comunicación en el que trabajan, utilizan la Red diariamente para buscar material para su trabajo, documentarse, contactar con fuentes, recibir o enviar información y verificarla. Si nos centramos en las fuentes 2.0 , los resultados obtenidos muestran que las más utilizadas son las redes sociales seguidas de lejos por los wikis, blogs y repositorios.

Mediante estas aplicaciones abiertas a la contribución colectiva de cualquier individuo, internet facilita el acceso a contenidos, ideas, etc., que de otro modo serían imposibles de alcanzar. No obstante, en ambos países los profesionales prefieren informarse y documentarse mediante fuentes reconocidas en internet, menos participativas y colaborativas, como son otros medios de comunicación o contactos personales, que les infunden más credibilidad que las fuentes 2.0. La excepción son las redes sociales, pero hay que destacar que ésta es la fuente que presenta más semejanzas con un contacto personal, al tratarse de una plataforma casi siempre de utilización personal donde los usuarios se presentan con su fotografía.

Aunque las redes sociales y los blogs sean las fuentes 2.0 más utilizadas en las rutinas periodísticas, los profesionales otorgan más fiabilidad a los repositorios y los wikis. Esta conclusión confirma una conexión entre el concepto de inteligencia colectiva, motor de wikis y repositorios, y la percepción de credibilidad de los periodistas con relación a estas fuentes de información.

\section{Se constata una mejora gradual con res- pecto a estudios anteriores en cuanto a la credibilidad de las informaciones en- contradas en redes sociales o blogs}

La insuficiente confianza depositada en algunas fuentes 2.0 sigue siendo el principal inconveniente en las redacciones: las dudas en cuanto a la exactitud y objetividad pueden originarse por la dificultad que tiene el periodista para identificar la autoridad, la actualización periódica y la cobertura, criterios más visibles en los wikis y repositorios, por ejemplo. Esta hipótesis podría explicar que este tipo de fuentes sean consideradas más fiables.

Aun así, se constata un aumento gradual de la credibilidad de informaciones encontradas en las redes sociales o los 
blogs con respecto a estudios anteriores. Este progreso puede ser debido a una mejor preparación de los periodistas en habilidades relacionadas con el uso de la Red, que les capacita para recuperar, buscar, identificar, seleccionar y verificar la información en este medio esquivando obstáculos como pueden ser informaciones anónimas, de mala calidad, incorrectas o falsas. Pero también puede estar relacionado con el hecho de que los periodistas sean conscientes de que las nuevas aplicaciones informáticas facilitan el funcionamiento de los distintos tipos de comunidades online y la vigilancia permanente que sus participantes ejercen para asegurar la fiabilidad de la información publicada.

\section{Nota}

1. Se utiliza la palabra "importante" para las cifras relativas a las respuestas "importante" y "muy importante".

\section{Bibliografía}

Asur, Sitaram; Huberman, Bernardo; Szabo, Gabor; Wang, Chunyan. Trends in social media: persistence and decay, 2011.

http://www.hpl.hp.com/research/scl/papers/trends/trends_ web.pdf

AccesoGroup. Estudio sobre el uso de la Red en los medios de comunicación. Madrid: Deloitte \& Touche, 2002.

http://www.pymesonline.com/formacion/index.php?action= file $\& i d=84$

Bastos, Helder. Jornalismo electrónico. Coimbra: Minerva, 1998.

Canavilhas, João. "Os jornalistas portugueses e a internet". En: $V$ Congreso Ibero-Americano de Jornalistas na Internet. Salvador da Bahia, 2004.

http://www.bocc.ubi.pt/pag/canavilhas-joao-jornalistasportugueses-internet.pdf

Cassidy, William P. "Online news credibility: an examination of the perceptions of newspaper journalists". Journal of computer-mediated communication, 2007, v. 12, n. 2, article 7. http://jcmc.indiana.edu/vol12/issue2/cassidy.html

David, Alicia; Glore, Peyton. "The impact of design and aesthetics on usability, credibility, and learning in an online environment". Online journal of distance learning administration, 2010, v. 13, n. 4.

http://www.westga.edu/ distance/ojdla/winter134/david glore134.html

Díaz-Noci, Javier; Meso, Koldobika; Larrondo-Urreta, Ainara; Salaverría-Aliaga, Ramón; Sadaba-Chalezquer, MaríaRosario. "Presencia y uso de internet en las redacciones de los diarios regionales vascos y navarros". Mediatika, 2010, n. 12, pp. 301-319.

http://www.euskonews.com/0383zbk/gaia38301es.html
Gans, Herbert. Deciding what's news: a study of CBS evening news, NBC nightly news, Newsweek and Time (25 $5^{\text {th }}$ anniversary edition). Evanston: Northwestern University Press, 2004. ISBN 9780810122376

Metzger, Miriam J. "Making sense of credibility on the Web: models for evaluating online information and recommendations for future research". Journal of American Society for Information Science and Technology, 2007, v. 58, n. 13, pp. 2078-2091.

http://www.ischools.org/conference08/pc/WC17_iconf08. $p d f$

http://dx.doi.org/10.1002/asi.20672

Masip-Masip, Pere. "Presencia y uso de internet en las redacciones catalanas". Zer, 2002, n. 14.

http://www.ehu.es/zer/zer14/presenciayuso14.htm

Masip-Masip, Pere. "Rutinas periodísticas e internet en la información diaria". En: Noves competències professionals. III Congrés intl comunicació i realitat, 2005, pp. 561-576. http://robertoigarza.files.wordpress.com/2008/11/conrutinas-periodisticas-e-internet-masip-2006.pdf

Noubel, Jean-François. Inteligencia colectiva, la revolución invisible, 2006.

http://solocreatividad.files.wordpress.com/2011/01/ intelco.pdf

Obercom. O jornalismo hoje: uma análise de 14 redacções de TV, rádio e jornais. Research report, 2006.

http://www.obercom.pt/client/?news/d=29\&fileName=rr1. $p d f$

Obercom. Desafios do jornalismo 2010. Lisboa: CIES. http://www.obercom.pt/client/?news/d=428\&fileName= desafios_do_jornalismo.pdf

O'Keefe, Daniel J. Persuasion: theory and research. Thousand Oaks: Sage, 2002. ISBN 9780761925392

Online News Association. Digital journalism credibility stu$d y, 2001$.

http://banners.noticiasdot.com/termometro/boletines/ docs/marcom/prensa/ona/2002/ona_credibilitystudy2001 report.pdf

Oriella PR Network. The state of journalism in 2011, 2011. http://orielladigitaljournalism.com/files/assets/downloads/ publication.pdf

Pavlik, John. Journalism and new media. New York: Columbia University Press, 2001. ISBN 9780231114837

Pinto, Manuel. "Fontes jornalísticas: contributos para o mapeamento do campo". Comunicação e sociedade, 2000, v. 14, n. 1-2, pp. 277-294.

http://repositorium.sdum.uminho.pt/bitstream/1822/ 5512/1/CS_vol2_mpinto_p277-294.pdf 